\title{
Mexeu com uma, mexeu com todas
}

When you mess with one woman, you mess all (of us!)

\author{
Ana Paula Saldanha Coelho \\ ORCID: https://orcid.org/0003-1276-8992 \\ Universidade Aberta, Portugal \\ E-mail: paula.coelho.mz@gmail.com \\ Carlota Inhamussua \\ ORCID: https://orcid.org/ 0002-3858-5038 \\ Grupo de Mulheres de Partilha de Ideias de Moçambique, Moçambique \\ E-mail: cmussua@gmail.com \\ Heike Friedhoff \\ ORCID: https://orcid.org/0002-9974-6288 \\ Grupo de Mulheres de Partilha de Ideias de Moçambique, Moçambique \\ E-mail: friedhoffheike@gmail.com
}

Article Info:

Article history: Received 2021-11-10

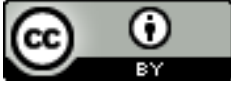

Accepted 2022-02-04

Available online 2022-02-04

doi: 10.18540/revesvl5iss2pp13848-01e

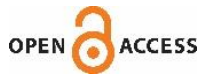

Resumo. O estudo destaca o papel dos movimentos sociais na defesa dos direitos da mulher rural enquanto fim e abraça a própria mulher enquanto objeto de um Movimento. Aborda que esse Movimento produz intervenção num contexto de desigualdade e numa realidade de vulnerabilidade. Este estudo qualitativo resulta da experiência e do trabalho de campo dos investigadores e da aplicação da metodologia de grupo de discussão. $O$ trabalho oferece os resultados que uma iniciativa de gestão feminista consegue produzir e destaca-a enquanto abordagem educativa que gera o empoderamento da mulher ajudando-a a colocar-se num espaço social e cultural no qual as normas consuetudinárias são desfavoráveis. O trabalho elenca que a aquisição de conhecimentos e aptidões e a Governação são importantes na construção da oportunidade da melhoria de vida da mulher rural. A experiência de atuação permite relatar que o Direito de Uso e Aproveitamento da Terra é limitado pelo espaço sociocultural e pelas redes existentes que criam constrangimentos no acesso aos recursos produtivos e à redução da pobreza. A experiência feminista contribui para ampliar o nível de consciência das mulheres, para ampliar o potencial que a disseminação de informação tem e para dar visibilidade à violação de direitos a que a mulher está exposta. O estudo apresenta a importância de trabalhar o meio rural e de empoderar a mulher por meio de instrumentos mais fortes ligados ao seu campo social. 
Palavras-chave: Género. Direitos das mulheres. Movimentos sociais. Meio ambiente. Terra. Governação.

Abstract. This research addresses the role of social movements in defending rural women's rights as an end and embraces women as the object of the Movement. Provides that this Movement produces intervention in a context of inequality and in a reality of vulnerability. This qualitative study is the result of the researchers experience and fieldwork and results from the application of a discussion group methodology. From the results of our study, we can say that a feminist management initiative produces very relevant results and is linked to an educational approach that generates the empowerment of women by helping them to place themselves in a social and cultural space in which cultural and social norms are unfavorable. This paper points out that the acquisition of knowledge and skills as well as Governance are important in building the opportunity to improve the lives of rural women. The field experience allows us to report that the Direito de Uso e Aproveitamento da Terra is limited by the sociocultural space and by the existing networks that create constraints accessing productive resources and to the reduction of poverty. The feminist experience contributes to increase the level of women's consciousness, to expanding the potential that the dissemination of information has and to provide visibility to the violation of rights to which women are exposed. As a contribution the research brings a critical insight about the importance of working on the rural area and on empowering women through stronger instruments linked to their social field.

Keywords: Gender. Women rights. Social movements. Environment. Land. Governance.

\section{Introdução}

Em Moçambique a pobreza e a desigualdade ainda constituem um grande desafio para as mulheres sendo um dos países com maiores níveis de desigualdade na África Subsahariana. Segundo o Relatório da Revisão Nacional Voluntária de Moçambique da Agenda 2030 para o Desenvolvimento Sustentável (2020) a disparidade entre os que se encontram em melhor situação financeira e os agregados familiares mais desfavorecidos está a crescer. A preocupação com a pobreza das zonas rurais do país leva-nos a centrar a atenção no potencial transformativo que a intervenção das e nas mulheres de base comunitária tem em termos de construção da mudança.

Estas mulheres de quem falamos vivem em comunidades que precisam de ser fortalecidas para que possam encontrar formas de requerer os recursos necessários para a sua vida e assim perseguirem os seus direitos. São mulheres com fraco acesso a bens e serviços, que têm acesso à terra embora desprovidas de mecanismos para a comercialização e conservação dos seus produtos cujas culturas muitas vezes se perdem.

Para analisar a forma de construir a mudança foi necessária a participação direta de investigadoras e ativistas por forma a recolher as perceções locais, e individualmente, das mulheres. A ajuda proporcionada às mulheres através do Movimento Social consubstanciou um dos objetivos da pesquisa, ensaiar como intervencionar a mulher rural pode gerar transformação. O segundo objetivo, observar a intervenção numa modalidade de gestão feminista dos recursos e, por último, 
identificar a importância da informação e do conhecimento na melhoria da vida dessa mulher rural.

Por ser a nossa pesquisa de cariz qualitativo, em termos de instrumentos de recolha de informação temos um percurso preliminar ligado à pesquisa documental $\mathrm{e}$ a entrevistas tendo sido daqui que se trabalhou o meio local rural através do instrumento grupo de discussão e do investigador-participante. Nestas referências é imperativo que liguemos os instrumentos aplicados a uma amostra não probabilística associada a critérios de escolha intencional sistematicamente utilizados (Carmo \& Ferreira, 2008). Em termos da dimensão da amostra que gerou os resultados apresentados assumimos que são generalizáveis apesar da existência de importantes diferenças entre normas e culturas, espaços sociais e culturais, que se encontram ao longo de todo o país, Moçambique, o que não dispensa a comprovação de cada realidade local e comunitária per si.

\section{Os Movimentos Sociais nos direitos da mulher rural}

A Constituição da República de Moçambique (2004), fala da "[...] edificação de uma sociedade de justiça social e a criação do bem-estar material, espiritual e de qualidade de vida dos cidadãos" (art. ${ }^{\circ} 11^{\circ}, \mathrm{c}$ ), da "[...] promoção do desenvolvimento equilibrado, económico, social e regional do país." (art. ${ }^{\circ} 11^{\circ}$, d) e diz que "[...] todo o cidadão tem o direito de viver num ambiente equilibrado e o dever de o defender" (art.. $\left.90^{\circ}\right)$.

Os movimentos sociais, enquanto objeto, definem-se como grupos de pessoas organizadas não institucionalizadas que defendem e representam em juízo ético e moral uma mesma causa e que advogam o conhecimento sobre direitos ou sobre a privação destes. Organizam-se de forma a gerar transformação, seja ela pessoal, coletiva ou a nível das estruturas. Estes conceitos são parte integrante do Movimento das mulheres que vamos apresentar.

Em Kant (1797) toda a ação tem um fim e "[...] o Fim é um objecto do livre arbítrio; porém, ninguém pode ter um fim sem se propor a si mesmo como fim do objecto do seu arbítrio". Ora, neste fim temos as mulheres no seu "direito de viver com dignidade e com a liberdade das carências" o que não se consegue sem o seu contributo para a "saúde e produtividade das famílias e comunidades inteiras, assim como para a construção de novas perspectivas para a próxima geração." (UNFPA, 2021).

Segundo Sainsaulieu (2001) as organizações constituem um espaço de produção e de relações económicas, um espaço de troca e de formação de valores e de modelos de conduta. A intervenção e a ação organizada das mulheres de base comunitária dá corpo a este espaço e dá visibilidade ao direito ao acesso e à posse da terra assim como à construção de alternativas para a mitigação dos efeitos das mudanças climáticas na sua vida e ainda à construção e ampliação da sua consciência para reiterarem os seus Direitos.

\section{O Grupo de Mulheres de Partilha de Ideias}

Em Moçambique, na Província de Sofala, o Grupo de Mulheres de Partilha de Ideias de Moçambique (GMPIS), fundado em 2014, constitui-se como um espaço dotado de movimento social, gerador de uma rede, baseado na solidariedade entre mulheres. Foi neste espírito de solidariedade que o GMPIS realizou uma campanha 
de solidariedade internacional para apoiar as mulheres e raparigas afetadas pelo ciclone IDAI.

Objetivamente, esta organização é uma experiência surgida em contexto de desigualdades, vivenciada por mulheres rurais, em diferentes partes de África e outras regiões do mundo, com avanços e distintos resultados a partir de uma construção solidária de respostas a situações de emergência em contexto de crise climática. Apresenta-se como uma Rede feminista de articulação, formada por mais de 30 organizações de base, pequenas organizações não-governamentais e mulheres ativistas, que trabalham em prol dos direitos das mulheres, a Rede GMPIS, está presente na Beira e em quase todos os 13 distritos da Província de Sofala, no Centro do país, como também mais recentemente nas Províncias de Gaza, Inhambane, Maputo e Cabo delgado.

Esta iniciativa surge após o IDAI, que devastou Sofala, para dar suporte a mulheres que sofreram o impacto do ciclone. Ventos fortes e inundações custaram a vida a centenas de pessoas e deixaram milhares sem base de suporte à sua existência. Segundo as agências humanitárias, mais de 500 mortos e 100 mil pessoas tiveram as suas vidas devastadas com "residências, estradas, escolas, infraestruturas de abastecimento de água e saneamento de dezenas de cidades" destruídas (OXFAM, 2020). A cidade da Beira, a segunda maior do país, ficou completamente submersa e isolada do restante país e milhares de pessoas ficaram sem acesso a serviços básicos em diversas localidades.

\subsection{A gestão feminista dos recursos}

Neste quadro de crise o Grupo de Mulheres, na iniciativa designada Mexeu com uma, mexeu com todas, angariou a solidariedade em vários cantos do mundo. Implementada por meio de uma plataforma digital de crowdfunding, registou doações de 8 países diferentes, direcionadas para o GMPIS. Na gestão desta iniciativa foram aplicadas estratégias, específicas, de comunicação e de atuação com o objetivo de dar transparência às ações realizadas no que concerne aos recursos financeiros doados e ao seu direcionamento. Tem de ser especialmente sublinhada uma gestão estratégica que destaca uma modalidade de gestão feminista dos recursos, que resulta do conhecimento específico e ímpar que a própria mulher tem das suas necessidades e das carências que avassalam o seu meio. Falamos de uma gestão que liga as ações realizadas a resultados, parte integrante de uma abordagem e atuação transformativas. Assim, uma abordagem que coloca e destaca a capacidade de fazer e de executar da mulher, geradora de empoderamento e que ajuda a desconstruir normas sociais vigentes do espaço sociocultural. Uma atuação estratégica que machamba [cultiva] novos conhecimentos e aptidões, portanto, uma oportunidade de melhorar a vida da mulher de base comunitária.

Em termos de alocação de recursos as mulheres ativistas de base comunitária criaram comissões para mapear o perfil das mulheres necessitadas e organizaram a compra e a entrega de produtos de primeira necessidade, alimentos e insumos agrícolas, como também material de construção. A atuação, em alguns casos, foi ainda mais longe com a construção de algumas casas para aliviar o sofrimento das mulheres e das suas famílias.

Complementarmente às entregas dos produtos foram realizadas reflexões feministas sobre o impacto das mudanças climáticas na vida das mulheres assim 
como sobre os direitos no acesso à terra. Além do trabalho humanitário foram realizadas ações de advocacia para desbloquear a ajuda para as beneficiárias. Identificaram-se casos em que a ajuda não chegava devido a aspetos ligados à cultura e às tradições patriarcais e ao posicionamento adverso de partidos políticos. Falamos de uma atuação efetivamente focada nas necessidades individuais que tem em conta as especificidades sociais e culturais do meio por forma a criar mudança e que expõe o necessário papel da Governação para a prossecução de fins sociais enquanto pedra angular na ação transformadora que pode exercer ao nível da mudança da vida da mulher rural.

Essa experiência interventiva através das mulheres de base comunitária trouxe resultados de curto, médio e longo prazo. Num curto espaço temporal foi possível a construção de uma agenda solidária que permitiu apoiar 726 mulheres que intervencionou 3.390 pessoas, ou seja, as famílias dessas mulheres, fortemente atingidas pelo IDAI. A médio prazo potencializou ações de advocacia sistemáticas que fortaleceram as agendas de resistência coletiva para a melhoria do acesso das mulheres à resposta a problemas causados por mudanças climáticas em alguns distritos da província de Sofala. Estas ações são um instrumento informativo, sobretudo educativo, importante pois "o desenvolvimento de conhecimentos e de competências representa um trunfo particularmente importante [...]. (por ser) um investimento em capital cultural que pode ser convertível em capital económico." (Feijó, 2015). Num horizonte temporal mais amplo, a longo prazo, nos resultados conta-se o fortalecimento do protagonismo do movimento de mulheres da região central de Moçambique, que atua conjuntamente desde 2015, e o fortalecimento da rede de solidariedade internacional.

Tudo o que referimos encontra importante expressão e é dotado de maior relevo quando através do relatório anual do Banco Mundial de 2019 - Erradicar a pobreza, investir em oportunidades - conseguimos corroborar a realidade descrita com a evidência das "barreiras à plena inclusão das mulheres nas economias e sociedades, juntamente com políticas que muitas vezes favorecem as elites em vez de gerar oportunidades de trabalho e apoio para os mais necessitados [...].". A formação de elites tem associada a desigualdade no acesso a recursos que geram vidas diferentes muitas delas não reconhecidas pelos atores sociais e que levam a que certos grupos, nomeadamente o rural, não sejam reconhecidos na distribuição [de recursos] criando assimetrias em cima de assimetrias. A atuação concertada e organizada do Grupo desencadeia a criação de um tipo de capital que gera condições através do conhecimento que permite questionar "O desafio existencial com que estamos confrontados" (NGOENHA, 2021).

\subsection{Direito à terra e empoderamento}

Falar dos efeitos desta tempestade e deste ciclone significa falar de uma população que já estava em sofrimento, desespero e extremamente vulnerável (UNICEF, 2021). Um impacto com elevada expressão na mulher moçambicana das localidades e dos postos administrativos. Projetamos que os movimentos sociais e as redes que a partir deles se criam têm importância na gestão ambiental sustentável, mas também no reforço do direito de posse e uso da terra. Estes movimentos, muito em especial o GMPIS, apesar de terem um âmbito nacional, constroem dinâmicas transnacionais - pois contam com o ativismo de feministas de diferentes nacionalidades - cujos fluxos internacionais mobilizam recursos, materiais e não materiais. Estamos em condições de afirmar que através do ativismo, ou seja, da 
efetivação deste movimento e Rede, questões como terra e meio ambiente, tão relevantes para a adequada e sustentável condição de vida da mulher, são variáveis endógenas de um sistema de empoderamento sustentável.

O discurso sobre mudanças climáticas muitas vezes é apresentado como neutro ao género e como discussão de um fato científico sobre um processo global, ao nível macro. Nos últimos anos, porém, ficou cada vez mais evidente que as mudanças climáticas têm um impacto diferenciado em mulheres e homens existindo evidências no mundo inteiro de que mulheres são mais afetadas e morrem mais e prematuramente por causa dos impactos. Estudos feitos em 141 países pela Escola de Economia de Londres mostram que entre 1981 e 2002, as calamidades e os seus impactos mataram em média mais mulheres que homens ou mataram mulheres mais cedo que homens $(\mathrm{GOH}, 2012)$. Num outro prisma de observação, não deixamos de anotar as mudanças comportamentais que se verificam nas mulheres tornando-as agentes para a mitigação das mudanças climáticas (WE EFFECT, 2019) que contribuem para a segurança alimentar, para uma dieta equilibrada com nutrição melhorada e contribuem para a redução da pobreza (WE EFFECT, 2017).

Ampliar o direito ao acesso e à posse da terra pode ser tido como um moto do Grupo. Em Moçambique, "a terra é propriedade do Estado e não pode ser vendida, ou por qualquer outra forma, alienada, hipotecada ou penhorada" (República de Moçambique, 2020). Grande parte da população, muito especialmente a mulher, depende da terra para viver, razão por que o "direito de uso e aproveitamento da terra é conferido às pessoas singulares ou coletivas tendo em conta o seu fim social" (ibidem). Esse direito é assegurado por meio de um instrumento jurídico chamado Direito de Uso e Aproveitamento da Terra, conhecido como DUAT. Contudo, são as relações desiguais de género associadas a fatores socioculturais que dificultam o acesso das mulheres à terra e aos recursos produtivos, por meio daquele Direito, reforçando e gerando as barreiras e os estereótipos de género.

Apesar de a mulher ter direito a requerer o DUAT vários fatores inibem o acesso a este documento do Direito. Nesta ocasião referimos um exemplo concreto das dificuldades geradas através dessas barreiras já que, ainda hoje, muitas mulheres não conhecem o DUAT nem estão conscientes sobre a importância de o reivindicar; outras mulheres, mesmo sabendo da sua importância, não possuem as condições exigidas por lei para garantir e adquirir o seu Direito. Essa situação provoca muitos impasses para as mulheres de base comunitária que vivem principalmente nas zonas rurais de Moçambique. Por tal motivo, a defesa do DUAT é estratégica nas lutas das mulheres rurais pelos seus direitos no país.

\section{A consciência de Direitos}

Esta experiência feminista moçambicana, baseada na problematização e reflexão, assim como no desencadear de ações coletivas, contribuiu para ampliar o nível de consciência das mulheres sobre os seus direitos no acesso à terra pelo que a podemos ligar, em complementaridade, ao processo educativo mencionado. As capacitações realizadas sobre o tema Direitos das mulheres à terra chegaram a agrupar 20 mulheres que depois deste processo educativo disseminaram 0 conhecimento em 5 Distritos onde tiveram lugar novos momentos educativos e de capacitação direcionados a outras mulheres rurais. 
Neste âmbito de atuação temática há mais resultados da atuação da Rede GMPIS para apresentar, nomeadamente o aumento do número de mulheres que compreendem como as questões ligadas à cultura e à tradição criam barreiras concretas para obterem o DUAT, especificamente a exigência de documentação e o pagamento de taxas inacessíveis. Ainda a anotar que as fortes influências das estruturas machistas dificultam o trabalho do GMPIS especificamente Chefes de Postos Administrativos que exercem o seu poder para a apropriação de casas construídas destinadas às mulheres e que foram ocupadas por homens. Outro resultado é a criação de ações coletivas pelas mulheres, no distrito de Búzi, para formalizar denúncias sobre a usurpação de direito de uso do DUAT por responsáveis da associação detentora do documento que incluía a parcela de terra das mulheres e a não abertura de uma cisterna e bomba de água para irrigação, que chegou a privar mais de 300 agricultores, na maioria mulheres, do acesso à água. Estes avanços e resultados favoreceram a ação para que o GMPIS tenha dado um passo adiante, consolidando ações de resistência e de advocacia específicas nomeadamente exigir do Governo a inclusão obrigatória do nome da mulher no DUAT no caso de casais. Essa agenda foi partilhada em vários fóruns da sociedade civil dentro do país e foi apresentada na Cúpula dos Povos da Southern Africa Development Community (SADC) na Namíbia, em 2018.

Da Estratégia de Género do Setor Agrário (República de Moçambique, 2005) retiramos que, no ano de 2005, $97 \%$ do total da terra arável era liderada por mulheres e chefes de família. Este fato coaduna-se com a existência de leis que objetivam facilitar o acesso das mulheres à terra. No entanto, as várias barreiras que se constituem como limitações induz a que as mulheres não consigam fazer valer os seus direitos. Este Movimento e estes ativismos das mulheres formam uma força de advocacia que lhes permite exigir tais direitos. Neste nosso caso estamos na presença de uma advocacia que se faz através de encontros nos quais as mulheres discutem e falam com os governantes e outros stakeholders com poder de influência e de decisão. Estamos a falar de uma advocacia de transformação já que são diálogos que transformam os líderes e esses por sua vez ficam sensíveis ao problema apresentado. Desta fase do ciclo de vida da advocacia criam-se as condições para que aconteçam mudanças no quadro legal que têm tanta mais força quanto mais as mulheres aperfeiçoarem a sua consciência crítica e fizerem ouvir a sua voz. Parece-nos importante realçar que esta é uma advocacia não confrontativa, uma advocacia de transformação que não exclui outras advocacias antes cria abertura para um processo construtivo e evolutivo.

Esta organização e governação do movimento social e da própria Rede de mulheres gera um conhecimento, um modo de atuar de apropriação global. Uma ativista lembra-nos que as pessoas nascem e crescem num meio em que a linguagem de direitos não existe e a consciência sobre o que é ser sujeito desses direitos numa circunscrição territorial figura como uma ausência. Esta realidade abre espaço à aceitação do que é cultural e tradicional levando à construção de entidades sociais. Para que uma nova linguagem, figuras sociais e direitos tenham espaço é impreterível que seja trabalhado o meio rural e nele tomar contato com as verdadeiras necessidades e realidades. A fundamentar esta abordagem invocamos Jane Parpat (2005) que defende o empoderamento dos mais pobres para a resolução dos seus próprios problemas já que mesmo na persistência da pobreza e no aumento indigno das desigualdades sociais (MOSCA, 2009) há uma mulher empreendedora que quer trabalhar e construir, mas que precisa de uma estrutura formatada que a apoie.

Apesar de haver caminhos a percorrer está criada a oportunidade de debate e de construção por meio desta experiência coletiva, de suma importância para a luta 
das mulheres rurais em Moçambique e, com certeza, em outros países. Na visão de Hahnemann (1921) um problema é permanentemente extinguido se se lhe afetar um instrumento mais forte que, a nosso ver, numa ação revitalizadora do campo social (BOURDIEU, 1984), leva a que a mulher rural, esclarecida e fortalecida, se posicione em defesa daquilo em que acredita.

\section{Conclusões}

Em guisa de conclusão, a mulher rural, consciente dos seus direitos, ouvida nas suas necessidades e participante ativa da estrutura social que integra, é construtora da solução para os seus problemas e ainda contribui positivamente para a comunidade em que está inserida. A informação e a advocacia contribuem para que a mulher rural tenha a possibilidade de sair de uma posição de subalternidade, dependente e sujeita, pois, fortalece-se, afirma-se e faz surgir um conjunto de oportunidades que constroem e remodelam uma forma de vida empoderada e menos condicionada por intervenções públicas que facilitam a acumulação dos interesses económicos dos grupos de interesse. Afirmamos que foi determinante a abordagem da análise qualitativa aos discursos das mulheres rurais assim como a participação direta em todos os processos de intervenção referidos para perceber que modalidade de atuação se pode constituir como um método para uma efetiva redução da pobreza e melhoria da condição de vida da mulher rural.

\section{Agradecimentos}

Os autores agradecem ao Grupo de Mulheres de Partilha de Ideias de Moçambique, à Coordenação e às Ativistas.

\section{Referências}

BOURDIEU, Pierre. Espace social et genèse des" classes". Actes de la recherche en sciences sociales, 52(1), 3-14, 1984.

CARMO, Hermano e FERREIRA, Manuela. Metodologia da Investigação Guia para a Auto-Aprendizagem. (2 ed.). Universidade Aberta, 2008.

FEIJÓ, João. Orientações culturais e recursos de poder-Relações laborais em Maputo. (1 $1^{\mathrm{a}}$ ed.). Maputo: Alcance Editores, 2015.

$\mathrm{GOH}$, Amelie. A literature review of the gender-differentiated impacts of climate change on women's and men's assets and well-being in developing countries. CAPRi Working Paper No. 106. Washington, D.C.: International Food Policy Research Institute, 2012. Disponível em: http://dx.doi.org/10.2499/CAPRiWP106. Acesso em: 10/01/2022

GOVERNO DE MOÇAMBIQUE. Direito do Uso e Aproveitamento de Terra. Portal do Governo de Moçambique, 2021. Disponível em: https://www.portaldogoverno.gov.mz/por/Cidadao/Informacao/Direito-do-Uso-e-Aproveitamento-de-Terra.

Acesso em:12/01/2022

HAHNNEMANN, Samuel. Organon of Medicine. (6th ed.). Jain Publishers (P) Ltd. P. 121-123, 1921

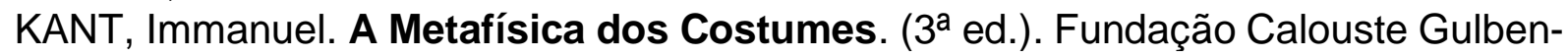
kian, 1797.

MOÇAMBIQUE, Elsa. Memórias do activismo: pelos direitos humanos das muIheres. Maputo, 2007. 
MOSCA, João. (2009). Economicando. (1 ${ }^{\mathrm{a}}$ ed.), Maputo: Alcance Editores, 2009.

NGOENHA, Severino Elias. O imperativo da desmosacanização, 2021, 20 de novembro. [Weblog]. Disponível em: https://www.severinongoenha.com/artigos/o-imperativo-da-desmosacanizao?mc cid=c13f59bf05\&mc eid=d978277399. Acesso em: 12/01/2022

ORGANIZAÇÃO DAS NAÇÕES UNIDAS. Declaração Universal dos Direitos Humanos, 1948.

OXFAM. Ciclone IDAI: $\mathbf{1}$ ano depois mais de $\mathbf{1 0 0}$ mil pessoas ainda sofrem os efeitos, 2020. Disponível em: https://oxfam.org.br/noticias/ciclone-idai-1-ano-depoismais-de-100-mil-pessoas-ainda-sofrem-os-efeitos/. Acesso em: 13/12/2021

PARPAT, Jane. Lessons from the Field: Rethinking Empowerment, Gender and Development in a Post (Post?) Development Perspective. In: WESSLING, Berndt Wilhelm (Org.). Feminist Post Development Thought: Rethinking Modernity, Post CoIonialism and Representation. London: Bloomsbury Academic, 2002

REPÚBLICA DE MOÇAMBIQUE. Relatório da Revisão Nacional Voluntária de Moçambique da Agenda 2030 para o Desenvolvimento Sustentável. Maputo: República de Moçambique, 2020

REPÚBLICA DE MOÇAMBIQUE. Perfil de Género de Moçambique. Ministério do Género, Criança e Acção Social. Maputo: República de Moçambique, 2016

. Estratégia de Género do Setor Agrário. Maputo: Ministério da Agricultura. Unidade de Género do MINAG, 2005.

de Moçambique, 2004.

Constituição da República de Moçambique. Maputo: República

Lei de terras 19/97, de 1 de outubro. Maputo: Ministério de agricultura. República de Moçambique, 1997.

SAINSAULIEU, Renaud. Sociologia da Empresa - organização, cultura e desenvolvimento. Lisboa: Instituto Piaget, 2001.

UNICEF. Ciclone Idai fustiga a Região Central de Moçambique, 2021. Mozambi-

que. Disponível em: https://www.unicef.org/mozambique/comunicados-de-imprensa/ciclone-idai-fustiga-regi\%C3\%A3o-central-de-mo\%C3\%A7ambique. Acesso em: $12 / 01 / 2022$

UNFPA. Igualdade de Género, 2021. Disponível em: https://mozambique.unfpa.org/pt/topics/igualdade-de-g\%C3\%A9nero. Acesso em: 10/01/2022

WE EFFECT. Triannual Report 2015-2017, The AGIR sub-programme in Natural Resources, Agriculture, Climate Change \& Environment - NACE. WE EFFECT, 2017.

Annual Report 2019, The AGIR sub-programme in Natural Resources, Agriculture, Climate Change \& Environment - NACE. WE EFFECT, 2019. 